\title{
Effects of laser acupuncture tele-therapy for rheumatoid arthritis elderly patients
}

\author{
Afnan Sedky Adly ${ }^{1,2} \cdot$ Aya Sedky Adly ${ }^{3,4}$. Mahmoud Sedky Adly $y^{5,6}$ \\ Received: 6 January 2021 / Accepted: 2 March 2021 \\ (C) The Author(s), under exclusive licence to Springer-Verlag London Ltd., part of Springer Nature 2021, corrected publication 2022
}

\begin{abstract}
Rheumatoid arthritis (RA) is a progressive common autoimmune disorder and is one of the most functional limiting diseases in elderly. Until recently, its treatment is mainly based on physical locations and meetings while being face to face. However, laser acupuncture tele-therapy approaches can significantly provide the patient with safety during the COVID-19 pandemic as well as changing the disorder's prognosis. Sixty patients were assigned randomly into 2 groups with 1:1 ratio. Patients in group A are treated remotely by laser acupuncture in addition to methotrexate and a tele-rehabilitation program in the form of aerobic exercise training. Patients in group B are treated by methotrexate and a tele-rehabilitation program in the form of aerobic exercise. There was a statistically significant difference in health assessment questionnaire (HAQ) pre- and post-treatment in group A $(p<0.05)$. The C-reactive protein (CRP) and interleukin-6 (IL-6) inflammatory markers as well as the malondialdehyde (MDA) oxidative marker showed a significant reduction pre- and post-treatment in group A $(p<0.05)$. Additionally, there was a significant increase in the adenosine tri-phosphate (ATP) antioxidant marker pre- and post-treatment in group A $(p<0.05)$. The comparison between groups A and B showed a statistically significant post-treatment difference in RAQoL, CRP, IL-6, ATP, and MDA in group A than group B. Considering the significant improvement that was found in the laser acupuncture group, it can be concluded that the use of laser acupuncture as adjunctive was effective in the treatment of elderly patients with RA. ClinicalTrials.gov Identifier: NCT04758689
\end{abstract}

Keywords Elderly $\cdot$ Tele-rehabilitation $\cdot$ Laser acupuncture $\cdot$ Rheumatoid arthritis $\cdot$ Geriatrics

\section{Introduction}

Rheumatoid arthritis is one of the most common and devastating autoimmune diseases that can cause progressive

Afnan Sedky Adly

afnansedky@yahoo.com

$1 \quad$ Faculty of Physical Therapy, Cardiovascular-Respiratory Disorders and Geriatrics, Laser Applications in Physical Medicine, Cairo University, Cairo, Egypt

2 Faculty of Physical Therapy, Internal Medicine, Beni-Suef University, Beni-Suef, Egypt

3 Faculty of Computers and Artificial Intelligence, Helwan University, Cairo, Egypt

4 Faculty of Engineering and Technology, Badr University in Cairo (BUC), Cairo, Egypt

5 Faculty of Oral and Dental Medicine, Cairo University, Cairo, Egypt

6 Royal College of Surgeons of Edinburgh, Scotland, UK disability and systemic inflammation. The global prevalence varies from 0.3 up to $1 \%$. Within 10 years of disease onset, at least $50 \%$ of the affected patients are limited functionally. Prevalence of comorbidities in elderly onset RA is higher than young onset RA due to the effects of aging process on the immune system. The diseaseassociated comorbidity prevalence can also be influenced by various genetic and environmental factors as it is considered a multifactorial disease $[1,2]$.

The elderly RA populations are obviously increasing; this can be attributed to the increasing life expectancy in elderly as well as the elderly onset RA increased incidence [3].

With aging the immune system deteriorates; this phenomenon is known as immunosenescence. Several studies revealed that the immune system of patients with RA undergoes premature and accelerated aging [4].

Elderly onset RA is characterized by a more systemic involvement leading to worse therapeutic outcome than the young onset RA [5]. 
Despite the expanding awareness of pharmacological agent adverse effects, management of RA depends mainly on the use of disease-modifying anti-rheumatic drugs (DMARDs), nonsteroidal anti-inflammatory drugs (NSAIDs), and biological agents. On the other hand, acupuncture has been widely used as a safe contemporary management option [6].

Acupuncture is recommended by the World Health Organization (WHO) for the treatment of sixteen inflammatory diseases including RA and more than thirty pain conditions [7].

Acupuncture can manage autoimmune diseases by regulating the immune network of the disease, exerting a regulating effect on the hemodynamic functions, and changing the concentrations of neurotransmitters in the central nervous system (CNS). The current evidence supports that the interaction between the immune and nervous systems is the main reason for the sensitization of the peripheral and central neurons leading to induction and maintenance of pathological pain [8].

According to Traditional Chinese Medicine (TCM) theory, RA is regarded as four syndromes including kidney and liver deficiency syndrome, blood stagnation syndrome, wind damp joint heat syndrome, and wind damp joint cold syndrome. Therefore, the systemic effects from the application of laser acupoints stimulation can make a great challenge in managing the disease $[9,10]$.

Concerns about adverse effects from therapeutic approach options can markedly influence the therapeutic decisions as patients as well as clinicians are often favoring a non-invasive and safe approach especially in this age group [11].

Despite the presence of these concerns, the therapeutic trials on this age group are limited due to the age-related high prevalence of comorbidities such as malignancies and infections [12].

Electronic health and tele-therapy solutions can provide better access to health and medical care. Their strengths made them crucial tools as a clinical response for the COVID-19 pandemic. Recent evidence shows that the tele-therapy can facilitate selfmonitoring and ensure patient involvement and adherence improvement, while giving higher value with a lower cost [13, 14].

Therefore, the aim of our study is to investigate a therapeutic approach targeting RA in elderly patients through teletherapy as it is considered safer and more challenging specifically in this group of patients in the presence of the pandemic.

\section{Patients and methods}

\section{Patients}

Sixty patients aged from 65 to 75 years of both sexes with active rheumatoid arthritis and not on DMARDs for 3 months participated in this study. The patients were 41 females and 19 males.

All patients were meeting the criteria of ACR and EULAR classification for rheumatoid arthritis [15]. Patients having malignancy and connective tissue diseases including systematic lupus erythematosus and systematic sclerosis, smokers, and alcoholics were excluded. The Cairo University research ethics approval was obtained before starting the study.

The sixty patients were randomly assigned into two groups with 1:1 ratio. The study group received laser acupuncture tele-therapy in addition to methotrexate and a telerehabilitation program in the form of aerobic exercise. The other group received methotrexate and a tele-rehabilitation program in the form of aerobic exercise, forming the control group. Written consent was obtained from all patients.

\section{The evaluation procedure}

The primary outcome measures for patients were IL-6, MDA, ATP, and CRP. While the secondary outcome measures for patients was RAQoL and HAQ.

The inflammatory markers including plasma interleukin-6 (IL-6) and C-reactive protein (CRP) was determined by ELISA methods. Oxidative marker of plasma malondialdehyde (MDA) and antioxidant marker of adenosine tri-phosphate (ATP) was determined through spectrophotometric methods.

Patients were asked to respond to the questions of rheumatoid arthritis quality of life (RAQoL) index which is concerned with unfulfilled requirements related to emotional well-beings, social interactions, and activities of daily living (ADL) that are relevant to patients with rheumatoid arthritis. Patients were also asked to respond to the questions of health assessment questionnaire (HAQ) which explores performance in twenty ADL areas [16, 17]. All measurements were taken pre- and post-treatment.

At the end of the treatment program, patients were instructed to fulfill a score sheet for the tele-therapy program. The score sheet involved the overall satisfaction, if they would recommend it to others, if it is convenient, if it is preferable, the quality of video and audio, if it is simple, and if they are generally convenient with the equipment.

\section{The clinical procedure}

Laser acupoints stimulation was established by a smart infrared laser system previously calibrated by the manufacturer with 808-nm wavelength, continuous wave $(\mathrm{CW})$, a power density of $100 \mathrm{~mW} \mathrm{~cm} \mathrm{~cm}^{2}$, energy density of $7.5 \mathrm{~J} \mathrm{~cm}^{2}$, and the irradiation period of $60 \mathrm{~s}$.

The system was capable of connecting and reading information from smart laser device as well as initiating the laser parameters and settings. This allowed the health professionals to control the smart laser device parameters and settings remotely.

An acupoint detector was used for specifying accurately the acupuncture points for each patient. The acupoints that were selected for laser radiation were Stomach (ST 36), Ququan (LR8), Yinlingquan (SP9), and Neiguan (PC6). The laser acupuncture sessions were undertaken in the period of 4 weeks with six sessions per week. 
In the study group, a laser acupuncture procedure was followed to produce a systemic effect relying largely on the immune-neuroendocrine network, which have been reported to be effective for different body organs [18].

Treatment with methotrexate was started at $15 \mathrm{mg}$ per week and gradually reduced by $2.5 \mathrm{mg}$ every 2 weeks for 4 weeks.

Aerobic exercise was in the form of treadmill walking at 3 mi per hour speed with fixed $0 \%$ inclination for $20 \mathrm{~min}$, three times weekly for 4 weeks.

\section{Tele-therapy and remote counseling}

The system architecture was designed with the ability to adopt the Internet of Things technologies. A simple application interface has been developed for communications between patients and health professionals in addition to storage and exchanging of medical information. The system facilitated for full-time technical supports to be available.

Once enrolled, the RA patients have installed the application on their phones and then went through a brief training concerning how the application would be used.

The application contains diagrams indicating the functions and locations of the acupuncture points, video scripts to demonstrate the proper probe holding technique, how to use the acupoint detector, and precautions when performing the laser acupuncture and/or aerobic exercises.

When providing the patient with the application, the patient was prompted to select the starting date for treatment.

Notifications and reminders were sent out to the health professionals in order to reply accordingly to the patient query. On the same way, the health professionals had the ability themselves for initiating a consultation according to outcomes of the periodical reports.

The system processed the online data as well as sending the patient data to the participating health professions after being analyzed (e.g., measurements were not in the normal range).

The system has also provided to the patients some frequent education messages about their health, questions and answers activity about RA, as well as some frequently asked questions. The system has a functionality that gives the ability to the healthcare professionals for accessing patients' measurements, data, and analysis. Patients have continued to receive usual care while being telemonitored via the system.

A videoconference meeting functionality was established to allow communication between the health professional and the patient via a communication client.

\section{Statistical analysis}

Changes in HAQ, RAQoL index, CRP, IL-6, ATP, and MDA were evaluated between the study and control group in addition to changes within the group by using one-way Anova with Tukey's multiple comparison test in order to decrease the possibility of type I error. The study data were tabulated, and statistical analysis was done by the Statistical Package for Social Sciences (version 19.0; SPSS Inc., Chicago, IL, USA) with a $p$-level below five percent to be considered significant.

\section{Results}

\section{Therapeutic outcomes}

As shown in Table 1, group A mean age was 68.87 with SD 2.69; however, group B mean age was 69.13 with SD 2.89 . The total mean age of the two groups was 69 with SD 2.8 .

Tables 2 and 3 showed the IL-6 and CRP inflammatory markers as well as the MDA oxidative marker, and there was a significant reduction pre- and post-treatment in group A, where $p$ value $<0.05$. Additionally, there was a significant increase in the ATP antioxidant marker pre- and posttreatment in group A, where $p$ value $<0.05$. Furthermore, there was a statistically significant difference in HAQ pre- and posttreatment in group $\mathrm{A}$, where $p$ value $<0.05$. The comparison between groups A and B post-treatment showed a statistically significant difference in IL-6, MDA, ATP, CRP, and RAQoL.

\section{Tele-therapy outcomes}

Twenty-six patients fulfilled the score sheets. In general, all of the patients that have responded were satisfied (value $=4.6$ ) and demonstrated that they would recommend the teletherapy program to others (value $=4.7$ ). Their feedback was unanimously demonstrating that the system was convenient, allowing open discussion, and made the access to a specialist possible to address their needs and concerns (value $=4.9$ ). The majority believed that it was very preferable than in-person consultation (value $=4.7$ ) and that it was simple (value $=4.3$ ). The patients were comfortable with the video quality (value $=$ 3.9 ) and the audio quality (value $=3.7$ ) and were generally convenient with the equipment (value $=4)($ see Fig. 1$)$.

\section{Discussion}

Traditionally, laser therapy and rehabilitation meetings between a patient and health providers have been carried out

Table 1 Mean and SD (standard deviation) of patients age by groups

\begin{tabular}{ll}
\hline Group & Age mean and SD \\
\hline Group A & $68.87 \pm 2.69$ \\
Group B & $69.13 \pm 2.89$ \\
Total & $69 \pm 2.8$ \\
\hline
\end{tabular}


Table 2 Comparative analysis of the primary outcomes

\begin{tabular}{llll}
\hline Primary outcomes & Tukey's multiple comparison test & Mean difference & Significance $(P<0.05)$ \\
\hline IL-6 & Group A (pre) vs group A (post) & 38.47 & $*$ \\
& Group A (pre) vs group B (pre) & -2.763 & $\mathrm{~ns}$ \\
& Group A (post) vs group B (post) & -41.00 & $*$ \\
MDA & Group B (pre) vs group B (post) & 0.2267 & $\mathrm{~ns}$ \\
& Group A (pre) vs group A (post) & 3.603 & $* * *$ \\
& Group A (pre) vs group B (pre) & 0.1111 & $\mathrm{~ns}$ \\
ATP & Group A (post) vs group B (post) & -3.344 & $* * *$ \\
& Group B (pre) vs group B (post) & 0.1472 & $\mathrm{~ns}$ \\
& Group A (pre) vs group A (post) & -44.52 & $* * *$ \\
& Group A (pre) vs group B (pre) & -0.6655 & $\mathrm{~ns}$ \\
Group A (post) vs group B (post) & 39.53 & $* * *$ \\
CRP & Group B (pre) vs group B (post) & -4.329 & $\mathrm{~ns}$ \\
& Group A (pre) vs group A (post) & 37.26 & $* * *$ \\
& Group A (pre) vs group B (pre) & 0.7527 & $\mathrm{~ns}$ \\
& Group A (post) vs group B (post) & -34.68 & $* * *$ \\
& Group B (pre) vs group B (post) & 1.826 & $\mathrm{~ns}$
\end{tabular}

$n s$ not significant, $p$ value $\geq 0.05$

$* * *$ Extremely significant, $p$ value 0.0001 to 0.001

*Significant, $p$ value 0.01 to 0.05 on physical locations while being face to face. This happens as a result of the fact that in many situations, tele-therapy is not being considered a stand-alone solution. Nevertheless, using non-invasive approaches enabled our study to consider teletherapy as being an alternative method for extending health professional support to the elderly communities who suffer from RA during the COVID-19 pandemic. In this study, the tele-therapy program provided new avenues for therapy and rehabilitation that gained an extensive acceptance from the patients and has been user-friendly.

The acupoints selected in this study are reported to induce systematic effects since several studies revealed that acupuncture can induce overall regulation involving multi-target, multi-link, and multi-level patterns [8].
It was reported in a study that stimulation of ST 36 with using anatomical tracers revealed that there is a specific relationship and connection between the central nervous system (CNS) and the ST 36. Additionally, it was demonstrated that acupuncture therapy at ST36 alleviated pain, reduced gastric dysrhythmia, and improved stomach emptying, which were mainly mediated by the neuro-transmitters pathways and opioids pathways [19].

Another study showed that acupuncture therapy at the Ququan (LR8) and Yinlingquan (SP9) acupoints increased the blood flow within the liver and spleen $[18,20]$. It was also found that the acupuncture therapy at the Neiguan (PC6) increased the cardiac functional parameters [21]. In addition, it was also confirmed that the liver is the
Table 3 Comparative analysis of the secondary outcomes

\begin{tabular}{llll}
\hline Secondary outcomes & Tukey's multiple comparison test & Mean difference & Significance $(P<0.05)$ \\
\hline RAQOL & Group A (pre) vs group A (post) & 2.733 & $\mathrm{~ns}$ \\
& Group A (pre) vs group B (pre) & -2.100 & $\mathrm{~ns}$ \\
& Group A (post) vs group B (post) & -4.533 & $* *$ \\
Group B (pre) vs group B (post) & 0.3000 & Non-significant \\
HAQ & Group A (pre) vs group A (post) & 0.2350 & $*$ \\
& Group A (pre) vs group B (pre) & 0.09167 & $\mathrm{~ns}$ \\
& Group A (post) vs group B (post) & -0.09733 & $\mathrm{~ns}$ \\
& Group B (pre) vs group B (post) & 0.0460 & $\mathrm{~ns}$ \\
\hline
\end{tabular}

$n s$ not significant, $p$ value $\geq 0.05$

**Very significant, $p$ value 0.001 to 0.01

*Significant, $p$ value 0.01 to 0.05 
Fig. 1 Patients scores (ranged between 0 and 5 , higher scores indicate better opinion)

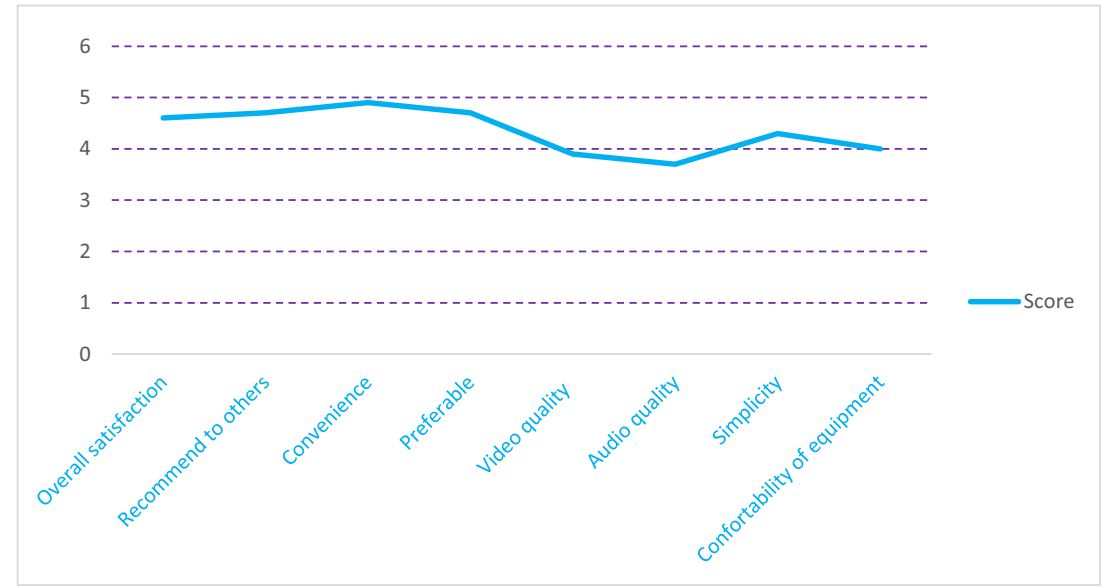

main source of the chemokine ( $\mathrm{C}-\mathrm{X}-\mathrm{C}$ motif) ligand 1 (CXCL1) in the blood immediately after application of acupuncture. This chemokine plays an important role in modulating pain through its analgesic effects. Acupuncture at ST36 was also found to produce stimulation of CXCL1 secretion in the liver [8].

It was also reported that stimulation at ST36 caused inhibition of nuclear factor kappa light chain enhancer of activated $\mathrm{B}$ cells $(\mathrm{NF}-\mathrm{kB})$ and significantly reversed the upregulation of its target gene p53 [22].

$\mathrm{NF}-\mathrm{KB}$ is considered an oxidative stress-responsive transcription factor, with well-documented involvement in ischemic injuries. Oxidative stress can promote the activation of $N F-\kappa B$ causing apoptosis and inflammation. Activated $\mathrm{NF}-\mathrm{KB}$ is able to mediate various gene transcriptions, and gene p53 is one of the important contributors to apoptosis in ischemic conditions [23].

Our study revealed that there was a significant reduction in MDA and a significant increase in ATP values indicating that oxidative stress was attenuated in the laser acupuncture-treated group. The mechanisms of action underlying this outcome may be related to the depression of calcium elevation and reduction of reactive oxygen species (ROS) generation which are mediated partially by the inhibition of NF- $\mathrm{KB}$ and consequently its target gene p53 activation [22].

The overall outcomes of the present study revealed that laser acupuncture caused remarkable anti-inflammatory and anti-oxidative effects. These results are similar to some recent studies which reported a reduction in the levels of the proinflammatory cytokines involving IL-6 and CRP $[12,24]$. In addition, it was also confirmed that acupuncture can regulate the body's own immune function through inhibition of immune cell communication and exertion of anti-inflammatory effect [6].

In this study, no significant improvement was observed in RAQoL, CRP, IL-6, ATP, and MDA in the control group B.
This indicates that the parameters and the selected acupoints for laser acupuncture were effective in improving function, inflammatory, and oxidative markers. The laser irradiation parameters of the study were selected based upon recent recommendations and have been adjusted remotely by the health professional [25].

The benefits of tele-therapy are providing local health services for elderly patients that may be particularly in great danger due to the COVID-19 pandemic as well as providing safety for the clinicians. Studies have found that there is increased susceptibility of infection by SARS-COV-2 in elderly patients. Also, the manifestations of COVID-19 may be masked in elderly patients taking disease-modifying antirheumatic drugs [26, 27].

It is still needed to assess laser acupuncture in different RA syndromes according to TCM theory. Selecting critical features for syndrome classification was not achieved in our study due to the limitations caused by the selected study design.

\section{Conclusion}

Our study provided an approach for how laser acupuncture tele-therapy can be used as a powerful alternative method to manage patients with rheumatoid arthritis by showing encouraging results.

Laser acupuncture tele-therapy was found to be an effective, convenient, and safe therapeutic approach by reducing inflammation and oxidative stress and improving quality of life and function in elderly patients having rheumatoid arthritis.

Acknowledgements We thank the participants of the study. We would like to thank Prof. Dr. Zahra M. H. Serry for her continuous encouragement and guidance. 


\section{Declarations}

Ethical approval All procedures performed were in accordance with the ethical standards of Faculty of Physical Therapy research ethics committee, Cairo University, and with the 1964 Helsinki declaration and its later amendments.

Informed consent Informed consent was obtained from all individual participants included in the study.

Conflict of interest The authors declare no competing interests.

\section{References}

1. Almutairi K, Nossent J, Preen D, Keen H, Inderjeeth C (2020) The global prevalence of rheumatoid arthritis: a meta-analysis based on a systematic review. Rheumatol Int. https://doi.org/10.1007/ s00296-020-04731-0

2. Organization WH (2020) Chronic diseases and health promotion: chronic rheumatic conditions. https://www.who.int/chp/topics/ rheumatic/en/. Accessed 2020

3. Safiri S, Kolahi AA, Hoy D, Smith E, Bettampadi D, Mansournia MA, Almasi-Hashiani A, Ashrafi-Asgarabad A, Moradi-Lakeh M, Qorbani M (2019) Global, regional and national burden of rheumatoid arthritis 1990-2017: a systematic analysis of the Global Burden of Disease study 2017. Ann Rheum Dis 78(11):1463-1471

4. Bauer ME (2020) Accelerated immunosenescence in rheumatoid arthritis: impact on clinical progression. Immun Ageing 17(1):1-14

5. Serhal L, Lwin MN, Holroyd C, Edwards CJ (2020) Rheumatoid arthritis in the elderly: characteristics and treatment considerations. Autoimmun Rev 19(6):102528

6. Chou P-C, Chu H-Y (2018) Clinical efficacy of acupuncture on rheumatoid arthritis and associated mechanisms: a systemic review. Evid Based Complement Alternat Med:2018

7. Organization WH (2013) WHO traditional medicine strategy: 2014-2023. World Health Organization

8. Xu Z-F, Hong S-H, Wang S-J, Zhao X, Liu Y-Y, Ding S-S, Xu Y, Zhang K, Yu N-N, Lu Z-X (2020) Neuroendocrine-immune regulating mechanisms for the anti-inflammatory and analgesic actions of acupuncture. World J Tradit Chin Med 6(4):384

9. Xie J, Feng H, Ding R, Dong W, Xin L, Liu J (2018) Risk factors for readmission of rheumatoid arthritis patients receiving integrative medicine: a retrospective analysis. Eur J Integr Med 20:200-205

10. Xie J, Li Y, Wang N, Xin L, Fang Y, Liu J (2020) Feature selection and syndrome classification for rheumatoid arthritis patients with Traditional Chinese Medicine treatment. Eur J Integr Med 34:101059

11. Adly AS, Adly AS, Adly MS, Mostafa YM (2018) Effectiveness of laser acupuncture as adjunctive therapy for limited range of motion in rheumatoid arthritis. Laser Phys 29(2):025601

12. Adly AS, Adly AS, Adly MS, Serry ZM (2017) Laser acupuncture versus reflexology therapy in elderly with rheumatoid arthritis. Lasers Med Sci 32(5):1097-1103

13. Adly MS, Adly AS, Adly AS (2020) Assessment of early orthodontic treatment on functional shifts by telemonitoring mandibular movements using a smart phone. J Telemed Telecare 26(3):150160
14. Adly AS, Adly AS, Adly MS (2020) Approaches based on artificial intelligence and the internet of intelligent things to prevent the spread of COVID-19: scoping review. J Med Internet Res 22(8): e19104

15. Aletaha D, Neogi T, Silman AJ, Funovits J, Felson DT, Bingham CO III, Birnbaum NS, Burmester GR, Bykerk VP, Cohen MD (2010) 2010 rheumatoid arthritis classification criteria: an American College of Rheumatology/European League Against Rheumatism collaborative initiative. Arthritis Rheum 62(9):2569 2581

16. Garrow AP, Papageorgiou AC, Silman AJ, Thomas E, Jayson MI, Macfarlane GJ (2000) Development and validation of a questionnaire to assess disabling foot pain. Pain 85(1-2):107-113

17. Pincus T, Keysor J, Sokka T, Krishnan E, Callahan LF (2004) Patient questionnaires and formal education level as prospective predictors of mortality over 10 years in $97 \%$ of 1416 patients with rheumatoid arthritis from 15 United States private practices. J Rheumatol 31(2):229-234

18. Li F, He T, Xu Q, Lin L-T, Li H, Liu Y, Shi G-X, Liu C-ZJPM (2015) What is the Acupoint? A preliminary review of Acupoints. Pain Med 16(10):1905-1915

19. Sun ZG, Pi YL, Zhang J, Wang M, Zou J, Wu W (2019) Effect of acupuncture at ST36 on motor cortical excitation and inhibition. Brain Behav 9(9):e01370

20. Yoon M-J, Kim S-Y, Park J-Y (2018) Recent study trends of the liver-tonification and liver-sedation of Saam acupuncture. Korean J Acupunct 35(1):1-17

21. Yang Q, Mao H, Chen X, Zhang Y, Zhang X, Liu Z, Jiang G, Huang WJM (2020) Neiguan (PC6)-based acupuncture pretreatment for myocardial ischemia reperfusion injury: a protocol for preclinical systematic review and meta-analysis. 99 (28)

22. Yang J-W, Wang X-R, Ma S-M, Yang N-N, Li Q-Q, Liu C-Z (2019) Acupuncture attenuates cognitive impairment, oxidative stress and NF- $\mathrm{kB}$ activation in cerebral multi-infarct rats. Acupunct Med 37(5):283-291

23. Kura B, Szeiffova Bacova B, Kalocayova B, Sykora M, Slezak J (2020) Oxidative stress-responsive microRNAs in heart injury. Int J Mol Sci 21(1):358

24. Seca S, Kirch S, Cabrita AS, Greten HJ (2016) Evaluation of the effect of acupuncture on hand pain, functional deficits and healthrelated quality of life in patients with rheumatoid arthritis - a study protocol for a multicenter, double-blind, randomized clinical trial. J Integr Med 14(3):219-227

25. Chon TY, Mallory MJ, Yang J, Bublitz SE, Do A, Dorsher PT (2019) Laser acupuncture: a concise review. Med Acupunct 31(3):164-168

26. Peron JPS, Nakaya H (2020) Susceptibility of the elderly to SARSCoV-2 infection: ACE-2 overexpression, shedding, and antibodydependent enhancement (ADE). Clinics 75

27. Sagnelli C, Gentile V, Tirri R, Macera M, Cappabianca S, Ciccia F, Coppola N (2020) Chronic conventional disease-modifying antirheumatic drugs masking severe SARS-CoV-2 manifestations in an elderly rheumatic patient. J Infect

Publisher's note Springer Nature remains neutral with regard to jurisdictional claims in published maps and institutional affiliations. 